Research Article

\title{
Impact of Modeling Simplifications on Lightning Strike Simulation for Aeroengine
}

\author{
Yi-fan Qian $(\mathbb{D}$, Zhi-feng Ye $\mathbb{B}$, and Hai-bo Zhang \\ Nanjing University of Aeronautics and Astronautics, Nanjing 210016, China \\ Correspondence should be addressed to Zhi-feng Ye; yzf@nuaa.edu.cn
}

Received 3 July 2019; Accepted 19 September 2019; Published 10 October 2019

Academic Editor: Rafał Stanisławski

Copyright ( 2019 Yi-fan Qian et al. This is an open access article distributed under the Creative Commons Attribution License, which permits unrestricted use, distribution, and reproduction in any medium, provided the original work is properly cited.

\begin{abstract}
With the development of electromagnetic simulation software and affordable hardware, it is allowed for us to complete simulations for EMC purposes. However, simulation demands will be immense when simulations for models with complex structures, especially aircraft components, have to be solved. Hence, it is meaningful to investigate how to minimize the computational demands. One of the solutions to reduce the simulation expense is the simplification for the simulated model. But the simplified model should be guaranteed to provide credible simulation results which do not deviate from the original model apparently. Generally, the difference between the simulation results and experimental data is estimated, or if the experimental conditions are not achieved, the comparison between the simplified model and the original one has to be analyzed, at least. This paper explores the electromagnetic simulation of a turbofan engine encountering lightning strike. With the simplifications of different components on the turbofan engine, the influences on induced currents of engine controller cables are simulated and analyzed based on the transmission-line matrix method. A combining method of components removal and geometric structure simplification is proposed to simplify the whole engine model. Simplified components include compressor, combustion chamber, turbine, and nozzle. The effects of different simplification methods are quantified, and the rationality of the simplified model is verified by simulation analysis.
\end{abstract}

\section{Introduction}

A commercial aircraft suffers lightning strike once a year on average in the world. The lightning impact can be divided into two categories: direct and indirect. Direct impact causes physical damage to the structure due to the direct contact of lightning. Indirect effects mainly refer to the transient changes of induced currents in cables of aircraft caused by electromagnetic effect of lightning strikes. These transient changes are caused by electromagnetic fields generated by lightning currents, which diffuse along the surface of aircraft through components such as windows or composites. In the interior of aircraft, these transients can exceed the immunity limit of the equipment, cause malfunction or damage, and endanger flight safety [1]. In order to solve the problem of lightning protection, electromagnetic simulation technology is increasingly applied to aircraft designs. Considering the lightning protection problem in the development stage of aircraft, it can reduce the cost of development and shorten the development cycle. Therefore, relevant research institutions have devoted large amount of manpower and material resources in the electromagnetic simulation technology of aircraft [2].

Aeroengine is the most significant part of aircraft. Once struck by lightning, if the shielding protection is weak, it will cause a devastating impact. In recent years, in order to investigate the electromagnetic protection problems often encountered by aeroengines in flight, various algorithms have been applied to such large-scale simulation. Da Silva and Bastos used the finite element method (FEM) to simulate and analyze large-scale electronic devices and simplified the simulation model [3]. Guiffaut and Reineix applied the finite-difference time domain method (FDTD) in the simulation of electromagnetic compatibility (EMC) for a small aircraft, and they analyzed the distribution of electromagnetic field for the aircraft under high-intensity radiation [4]. High-precision simulation can provide guidance for the design, certification, and life cycle prediction of 
aeroengine, thus improving flight safety. At the same time, with the increasing computing power of calculating machine, it is possible to simulate the electromagnetic effect for the whole aeroengine structure. However, the electromagnetic simulation of such large complex structures is a timeconsuming process, and the calculation process is arduous. For the lightning strike simulation of aeroengine, the calculation time of the simulation can be even up to several weeks. It is definitely meaningful to reduce the cost of such simulations so that the development cycle can be shortened [5]. Recently, the development of electromagnetic simulation software and affordable hardware allows scholars to complete simulations for large-scale structures. But electromagnetic simulation demands are still enormous for a model with complex structures, especially aeroengine. Hence the need to minimize the computational demands is worthy of investigation. One of the feasible methods to reduce the computational expense is the simplification for the original model. But the simplified model should be guaranteed to provide credible results of simulation. Generally, the comparison of the simulation results with experimental data is estimated. Actually, if there are no experimental data, it is necessary to analyze the difference of simulations between the simplified model and the original one. Therefore, a simplification method is urgently needed to reduce the overall cost and the required time of simulation. In the past 10 years, a few scholars began to discuss the simplification methods of such kinds of simulation, gradually. The existing simplification methods mainly focus on the details and parts of the electromagnetic simulation model. Arnaud Christophe of Fiat Automobile Companys in Brazil has roughly classified the structural components of an automobile model, the components were removed and simulated one by one, and comparison between the effects of different simplified classifications on the electromagnetic field intensity in the vehicle was obtained [6], but the model is simple so the revivification is low. Reznicek and Raida of EVEKTOR Aircraft Manufacturing Company in Czech simulated the electromagnetic field of its small-sized turboprop aircraft, taking the electromagnetic field intensity in the cabin as the simulation target, some parts of the aircraft were removed and simplified. The simulation results after removal and simplification were compared, and the rationality of geometric simplification in electromagnetic simulation was verified [7]. However, effects of induced currents in cables caused by lightning strike were not studied. Engineer Guadalupe Gutierrez et al. of Airbus simplified the three components in the structure of a turboprop loaded by A400M transporter, i.e., the air particulate separator, the oil cooler, and the electric contact. The effects of simplification on the induced currents in the internal cables of turboprop were studied in detail to prove the rationality of simplification [8]. The peak values of the induced currents were taken as the judgment in this paper with the overall error of currents.

This paper is organized as follows. Section 2 provides the simplification method of aeroengine, Section 3 shows the modeling process of aeroengines and cables, simulation configurations are presented in Section 4, and comparison and analysis are shown in Section 5. Finally, simulation scheme and verification are presented in Section 6. In Section 7, we draw the conclusions briefly.

\section{Simplification Method}

The primary task of geometric simplification is to define the simulation target. For an aeroengine, the largest impact of indirect lightning strike effect is that the transient current in the cable exceeds the immunity of the aeroengine controller, which leads to the malfunction and causes potential safety hazards. Secondly, the establishment of simulation model is also very significant. The higher the accuracy of structure and cable layout, the higher the simulation revivification, and the more complex the model details are, thus, more detailed grouping and simplification of components can be carried out. Although simplification is an important way to reduce simulation cost, the rationality of simplification is also needed to be guaranteed. Each simplified simulation should be compared with the simulation results of the original model, and the data should be analyzed in detail. The simplification methods of components are classified (removal, detail processing, and cannot be removed), so as to guarantee the rationality for simplification. For the electromagnetic simulation of a whole aeroengine structure, whether the model is simplified reasonably or not will greatly affect the accuracy of the final results, otherwise the simulation will be meaningless. The flow chart is shown in Figure 1.

\section{Modeling}

3.1. Geometric Structure. A certain turbofan engine is built as the research object. The model structure includes nacelle, inlet, compressor, combustor, part of fuel lines, turbine, and nozzle. The total length is about 4 meters, as shown in Figure 2.

3.2. Modeling of Cables. Four cables are established in the model according to the real position of the wiring harnesses in the turbofan engine. Different areas are covered by the cables imitating the connection with few major components of the aeroengine, as shown in Figure 3.

In order to show the effect of various types of cables, the harnesses include four types of cables which are most commonly used in aeroengines [9], namely shielded twisted pair, twisted pair, double-shielded twisted pair, and coaxial line, respectively. Table 1 lists the details of the cables.

3.3. Components Grouping. The influence of induced current in the cables for different components is the focus of this paper. Because the existence of a nacelle has a great impact on the electromagnetic shielding ability of the engine, it cannot be removed, and it is not considered when grouping. Therefore, several main components of the engine are divided into nine groups, regarding the whole engine model as one group, there are ten groups in total, and their English name and abbreviation are shown in Table 2. 


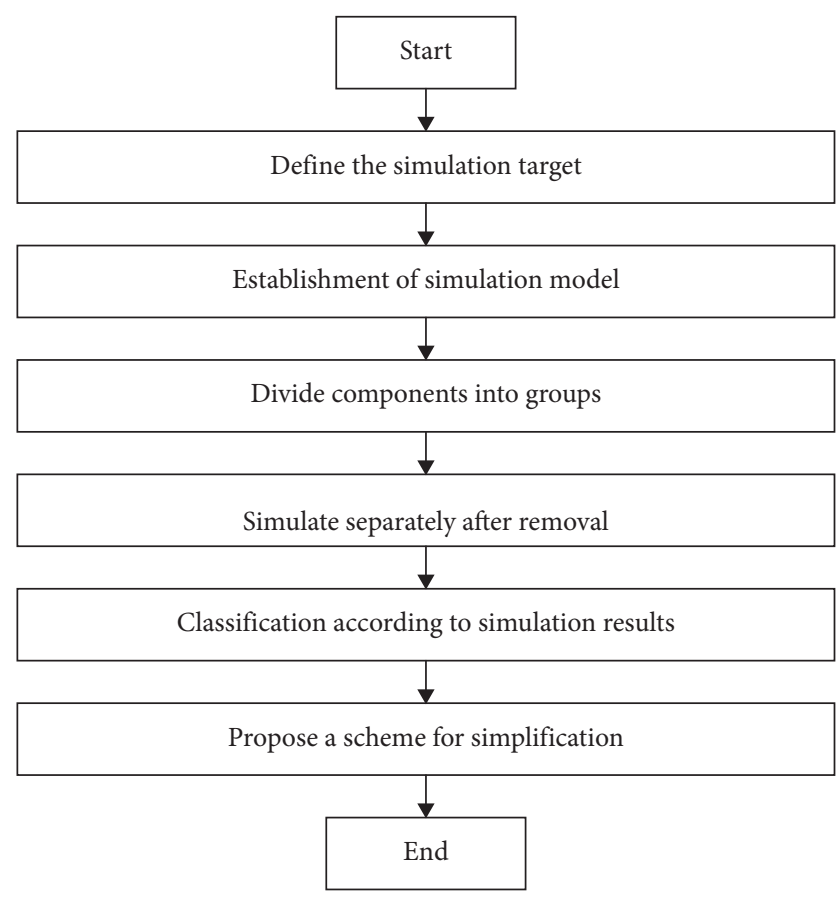

Figure 1: Flow chart of simplification.

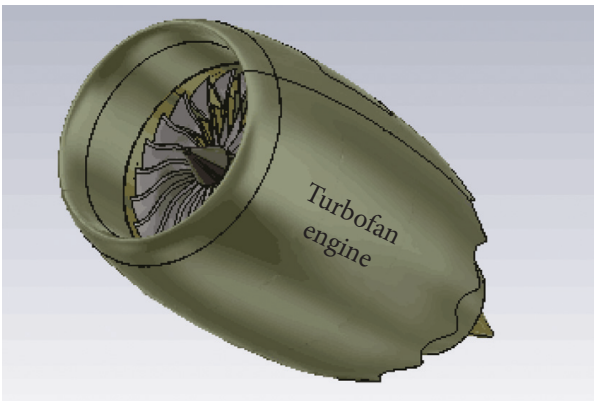

Figure 2: 3D model of turbofan engine.

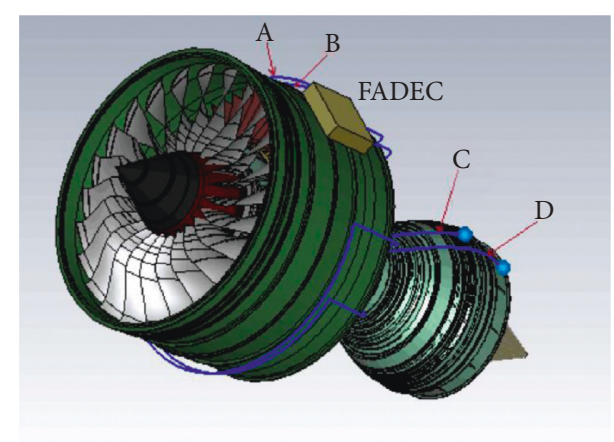

Figure 3: Diagram of internal cables.

\section{Simulation}

In this paper, CST software based on the transmission-line matrix method (TLM) is used to simulate the electromagnetic effect of lightning strike on a turbofan engine. The simulation conditions are set according to the current
TABLE 1: Information of cables.

\begin{tabular}{l} 
Cables $\quad$ Areas \\
\hline A Compressor \\
B Shielded twisted pair \\
Combustor \\
Twisted pair \\
C \\
Turbine \\
Double-shielded \\
twisted pair \\
Coaxial line \\
Nozzle
\end{tabular}

TABLE 2: Component grouping of aeroengine.

\begin{tabular}{lc}
\hline Groups & Abbreviations \\
\hline Aeroengine model & ALL \\
Fan & Fan \\
Intermediate-pressure compressor & IPC \\
High-pressure compressor & HPC \\
Fuel nozzle & FN \\
Combustor & Combustor \\
High-pressure turbine & HPT \\
Intermediate-pressure turbine & IPT \\
Low-pressure turbine & LPT \\
Nozzle & Nozzle \\
\hline
\end{tabular}

waveform and lightning test method defined in SAE ARP 5412 [10] and SAE ARP 5416 standards [11].

4.1. Transmission-Line Matrix. The transmission-line matrix method (TLM) is used to solve the three-dimensional timedomain problem of Maxwell equation, in which the transmission line is a facility capable of transmitting electromagnetic energy between two points with minimum radiation. In the TLM method, firstly, the engineering problem is simulated through the continuous transmission 
line network, each node in the network is represented by a lumped element, whose physical parameters correspond to the actual problem, i.e., spatial discretization. The lumped element is simulated by each node of the transmission line, i.e., time discretization. One-dimensional transmission line is taken as an example, and its equivalent circuit is shown in Figure 4.

According to Kirchhoff's law, we have

$$
\left\{\begin{array}{l}
\frac{\partial^{2} V(z, t)}{\partial z^{2}}=l c \frac{\partial^{2} V(z, t)}{\partial t^{2}} \\
\frac{\partial^{2} I(z, t)}{\partial z^{2}}=l c \frac{\partial^{2} I(z, t)}{\partial t^{2}}
\end{array}\right.
$$

where $V$ and $I$ stand for the voltage and current in the circuit, $z$ is the position coordinate, $t$ is the time coordinate, and $l$ and $c$ represent the distributed inductance and capacitance parameters, respectively.

The two-dimensional TLM is composed of parallelconnected transmission line grids. Four pulses incident to a node from four branches, scattering first, and then incident to adjacent nodes, respectively. It can be expressed as

$$
\begin{aligned}
{ }_{k+1} V^{i} & =C_{k+1} V^{r}, \\
{ }_{k+1} V^{r} & =S_{k} V^{i},
\end{aligned}
$$

where $V^{i}$ is the excitation voltage matrix, $V^{r}$ is the reflection voltage matrix, $S$ stands for the impulse scattering matrix of the node, $C$ is the connection matrix describing the network topology, and subscripts $k$ and $k+1$ represent the discrete time interval of scattering. Thus, the scattering formula can be obtained as follows:

$$
{ }_{k+1} V_{n}^{i}=\frac{1}{2}\left[\sum_{m=1}^{4}{ }_{k} V_{m}^{i}\right]-{ }_{k} V_{m}^{i},
$$

where $r$ stands for scattering, $i$ stands for incident, and $m$ and $n$ represent the port numbers, respectively.

The three-dimensional TLM is based on a symmetrical condensation node (SCN) algorithm to simulate the electromagnetic propagation in the space unit. Each three-dimensional SCN has six branches, one branch is composed of two vertical transmission lines to simulate the propagation of electromagnetic field. Because the slot model can be regarded as the dual form of a dipole antenna, according to the Babinet theorem, the radiation field vector on the slot is the same as the equivalent dipole, but the electric and magnetic field vectors are interchangeable. For the narrow slot model, the field along the long edge of the slot forms an interface through a one-dimensional transmission line connected with a symmetrical condensation node, and it synchronizes the slot with the residual pulse and the time step of the three-dimensional TLM unit through the capacitance and inductance of the slot unit length, thus the global scattering model is established [12]. The formulation is as follows:

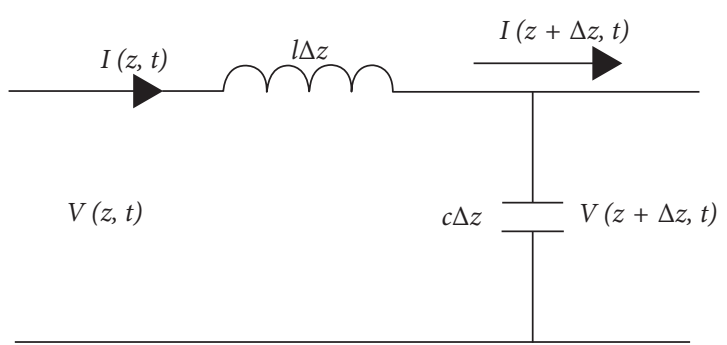

FIgURE 4: Lumped-parameter model of transmission line.

$$
\begin{aligned}
Y_{L} & =\frac{\Delta t}{L_{s} \Delta l} \\
Y_{s} & =2\left(\frac{C_{s} \Delta l}{\Delta t}-Y_{L}\right) \\
C_{s} & =0.637 \varepsilon \ln \left(\frac{0.563 \Delta l}{\omega}\right)+\frac{\varepsilon d}{\omega} \\
L_{s}^{-1} & =\left[\frac{\pi \mu}{2 \ln (1.591 \Delta l / \omega)}\right]^{-1}+\left(\frac{\mu \omega}{d}\right)^{-1} .
\end{aligned}
$$

In the formula, $Y_{L}$ is the characteristic admittance of the transmission line, $Y_{S}$ is the capacitive characteristic admittance, $\varepsilon$ is the relative permittivity in free space, $\mu$ is the permeability, and $\Delta l$ is the distance of electromagnetic wave transmission.

The TLM can deal with complex nodes, simulate wave propagation in time domain, simplify calculation, and avoid possible problems such as convergence, stability, and pseudosolution. The TLM can also get the main- and highorder mode eigenvalues through the Fourier transform, which can accurately simulate the physical characteristics and behavior of waves and can explain the propagation characteristics of different states. Therefore, it is very suitable for the simulation of three-dimensional time-domain electromagnetic field [13].

4.2. Excitation Source. In this paper, the expression of lightning impulse excitation waveform of airborne electronic and electrical system is $I(t)=I_{0}\left[e^{-\alpha t}-e^{-\beta t}\right]$, $I_{0}=218810 \mathrm{~A}, \alpha=11354 \mathrm{~s}^{-1}$, and $\beta=647265 \mathrm{~s}^{-1}$, which is given by SAE ARP 5412 and SAE ARP 5416 standards. Lightning strike point is set at the axle tip of the turbofan engine which is most likely to be struck by lightning [14], and the exit point of lightning strike signal is at the end of the nozzle, as shown in Figure 5. The boundary condition is set to "open" to simulate the infinite free space which means electromagnetic waves will be absorbed completely without any reflection when they hit these boundaries. The waveform of the excitation signal is shown in Figure 6. The peak current is $218.81 \mathrm{kA}$, peak time is $6.4 \mu \mathrm{s}$, and duration is $500 \mu \mathrm{s}$; frequency is set at $0-50 \mathrm{MHz}$, and there is almost no current component above $50 \mathrm{MHz}$. It can represent the most extreme lightning strike situation and save time [15]. 


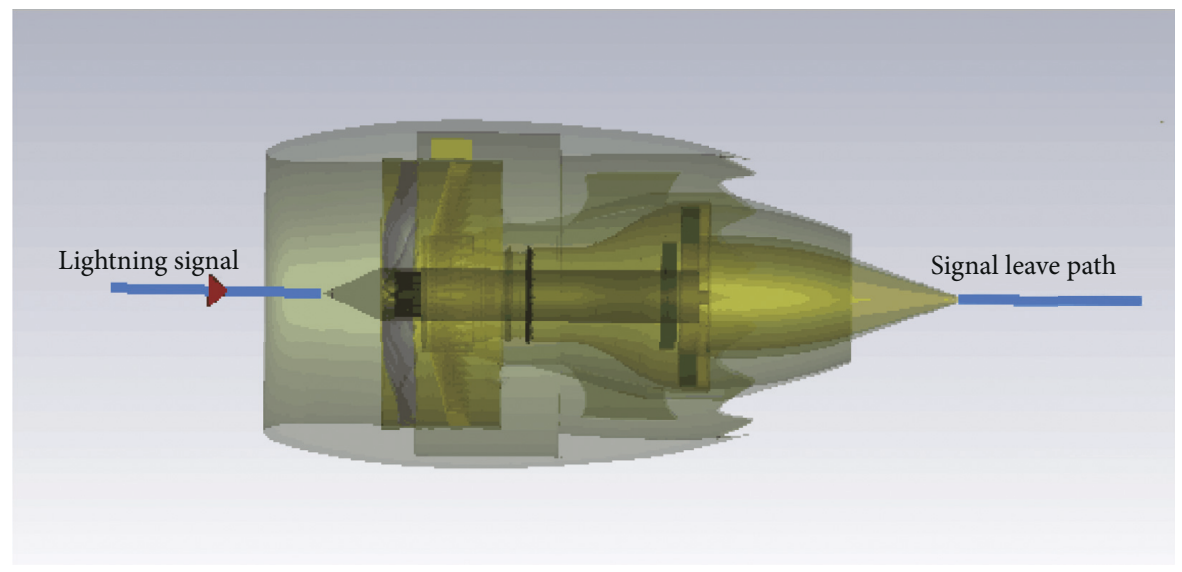

FIGURE 5: Lightning strike point.

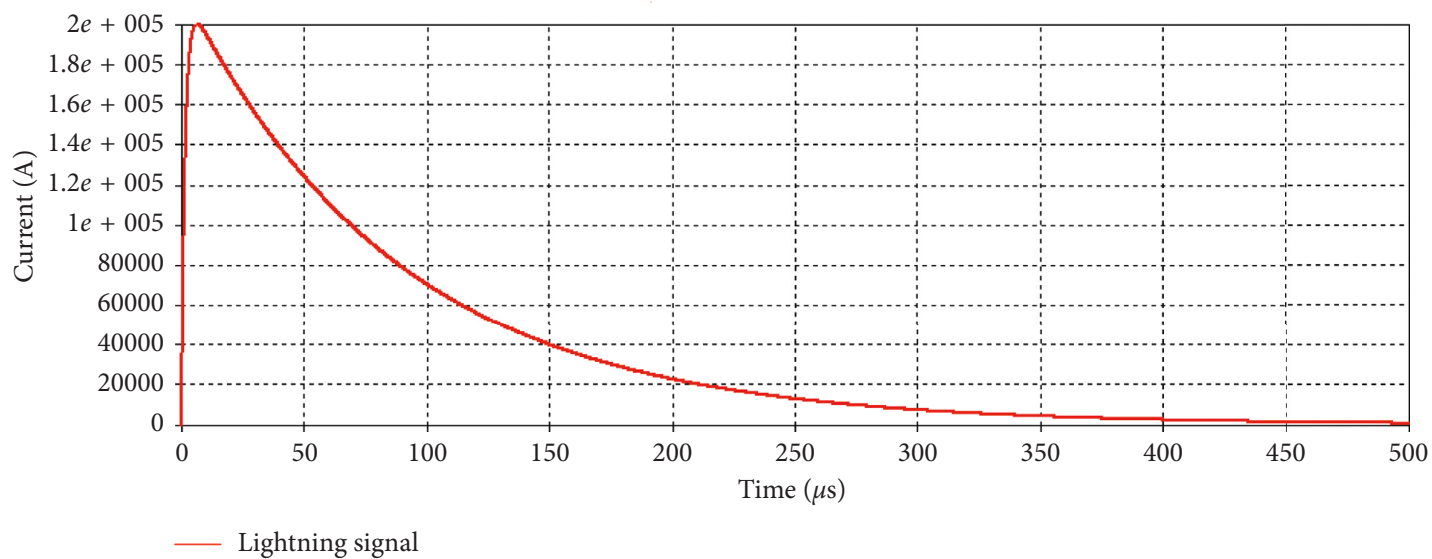

FiguRE 6: Lightning signal waveform.

The schematic diagram is shown in Figure 7, in which the yellow part stands for the lightning signal, the resistance between twisted pairs is $100 \Omega$, the matching resistance of coaxial cable is $50 \Omega$, and the shielding layers are all grounded.

\section{Simulation and Analysis}

5.1. Simulation. Each cable is equipped with a probe to measure the induced current, according to the groups divided from the components in Section 3.2, and the simulation results of the models after removing each group are obtained. Figures 8-11 show the simulation results of four cables. The abscissa is time, the unit is $\mu$, the longitudinal coordinate is induced current, and the unit is A. As can be seen from Figures 8-11, compared to the curves of the original model, the induced current corresponding to the removal of some components has changed considerably, such as fan (Fan) and intermediate-pressure compressor (IPC). The simulation results of the induced current corresponding to some simplified models are similar to those of the original model, such as nozzle (Nozzle) and low-pressure turbine (LPT).
5.2. Data Analysis. In the previous section, the induced current data in each cable of the simplified model has been obtained after removing different components. In order to obtain the quantified results, the simulation results of the original model are taken as the standard values. The data of each simplified model is compared with the data of the original one. Finally, the influence of the simplified components for the induced current in cables is obtained. Because the peak value of induction current of various types of cables differs greatly, some scholars have used decibel error to measure the error in the study of induction current errors. This analysis method is worth for reference [16]. Therefore, the decibel error in electronic instrument measurement and the root mean square error (RMSE) in mathematical statistics are used. In numerical calculation, the decibel error represents the difference between the accurate value and the approximation, that is, the error between the simulation result $J_{i}$ of the original model and the simulation result $I_{i}$ of the simplified model. For the induced current in a single cable, the expression is as follows:

$$
\xi_{i}=20 \lg \left(\frac{I_{i}}{J_{i}}\right) \mathrm{dB}, \quad i=1,2, \ldots, n,
$$




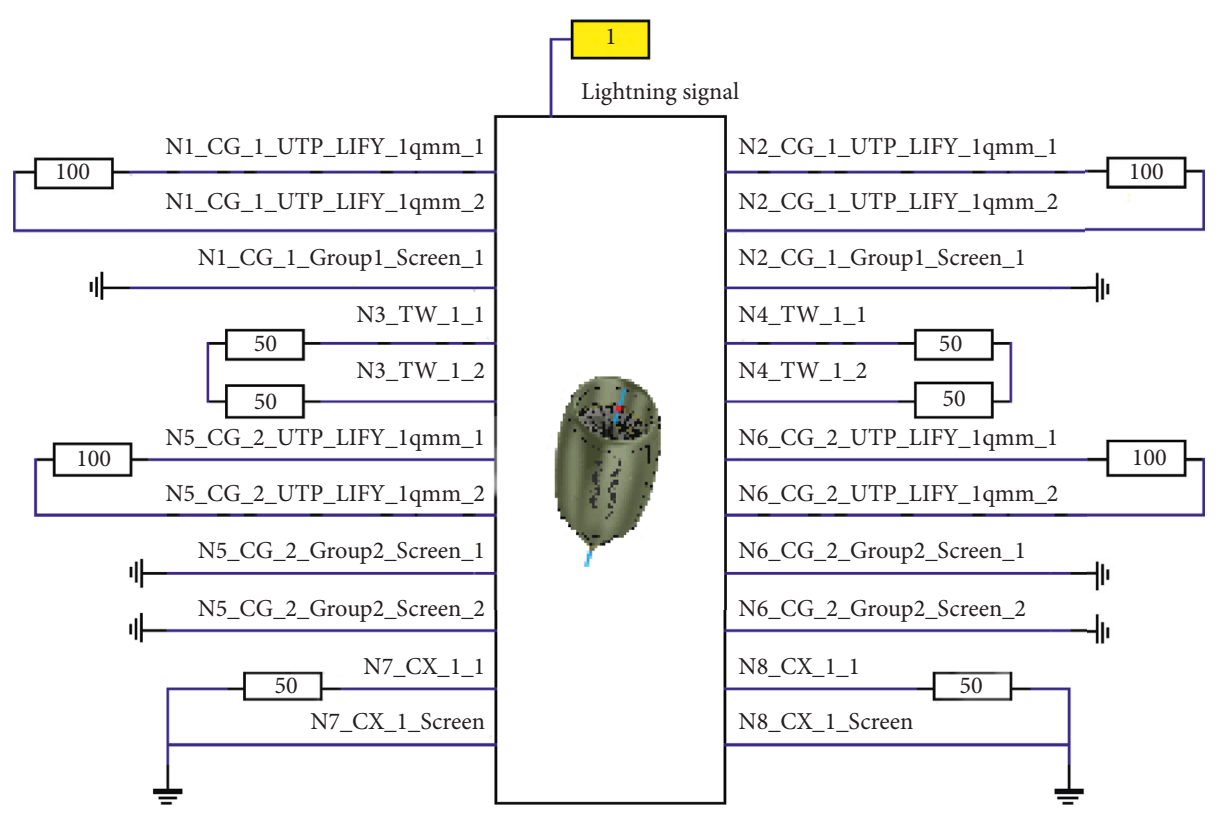

FIgURE 7: Schematic diagram.

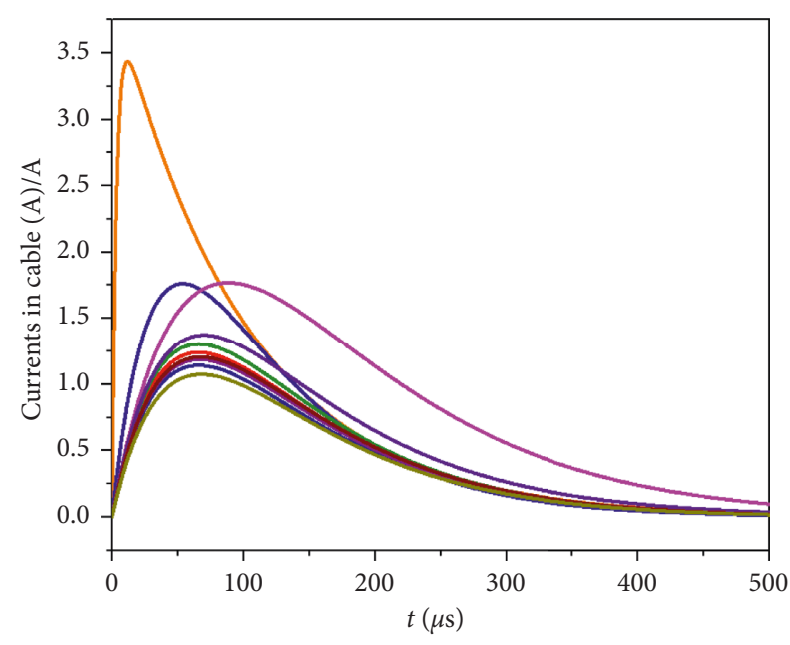

Simplified models

$\begin{array}{ll}\text { - ALL } & - \text { Combustor } \\ \text { Fan } & - \text { HPT } \\ \text { IPC } & - \text { IPT } \\ \text { HPC } & - \text { LPT } \\ \text { FN } & - \text { Nozzle }\end{array}$

Figure 8: Comparison of simulation results on Cable (A).

where $n$ stands for the sampling time points.

The RMSE is used to measure the overall situation of the error, and its expression is as follows:

$$
\gamma_{i, j}=\sqrt{\frac{1}{n} \sum_{k=1}^{n}\left(\xi_{i, j, k}\right)^{2}},
$$

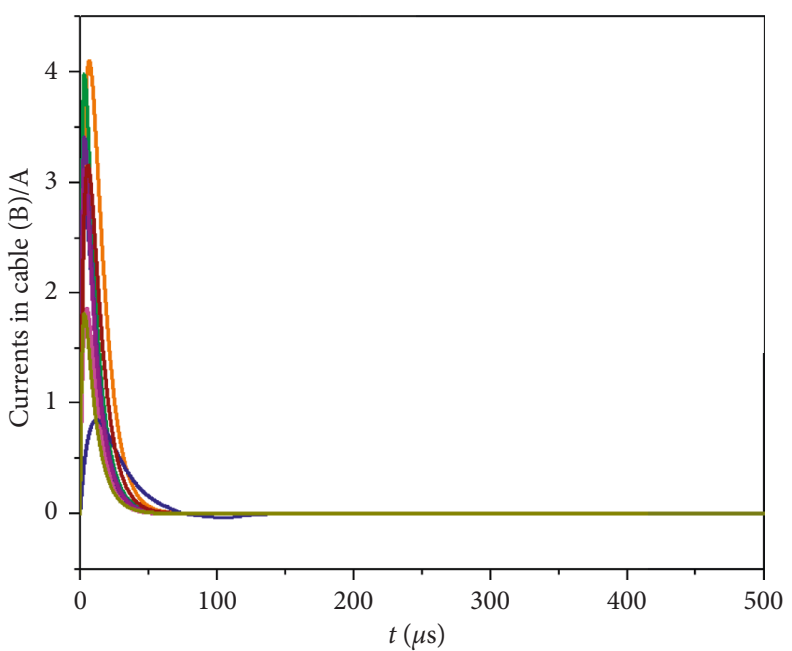

Simplified models

$\begin{array}{ll}\text { - ALL } & - \text { Combustor } \\ \text { Fan } & - \text { HPT } \\ \text { IPC } & - \text { IPT } \\ \text { HPC } & - \text { LPT } \\ \text { FN } & - \text { Nozzle }\end{array}$

Figure 9: Comparison of simulation results on Cable (B).

where $\gamma_{i, j}$ represents the RSME of the decibel error in the cable $j$ with the removal of group $i$ in the simulation, which denotes the effect of $i$ group on the induced current in cable $j, n$ stands for the number of samples of the induced current, and $\xi_{i, j, k}$ represents the decibel error of the induced current at the $k$ sampling point on cable $j$ and the corresponding 


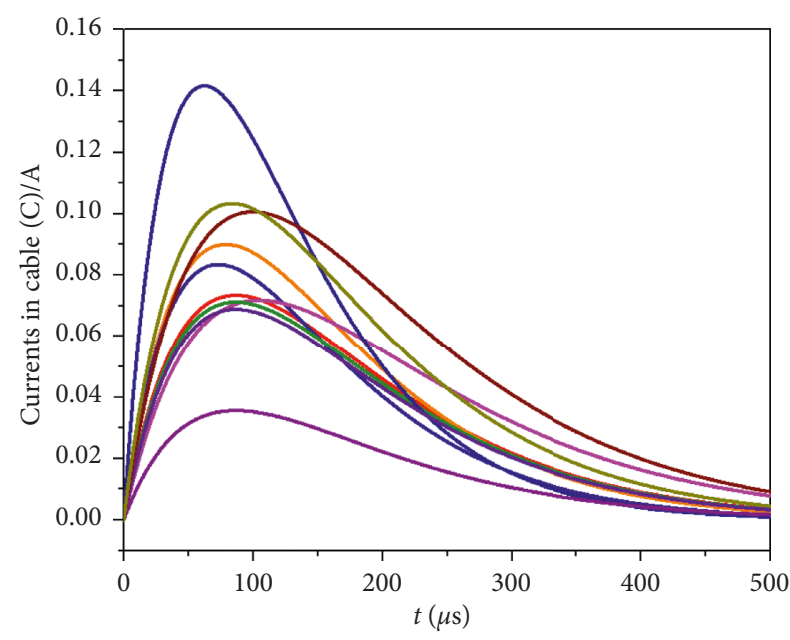

Simplified models

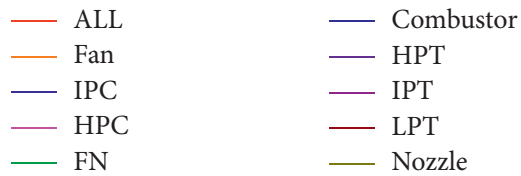

FIGURE 10: Comparison of simulation results on Cable (C).

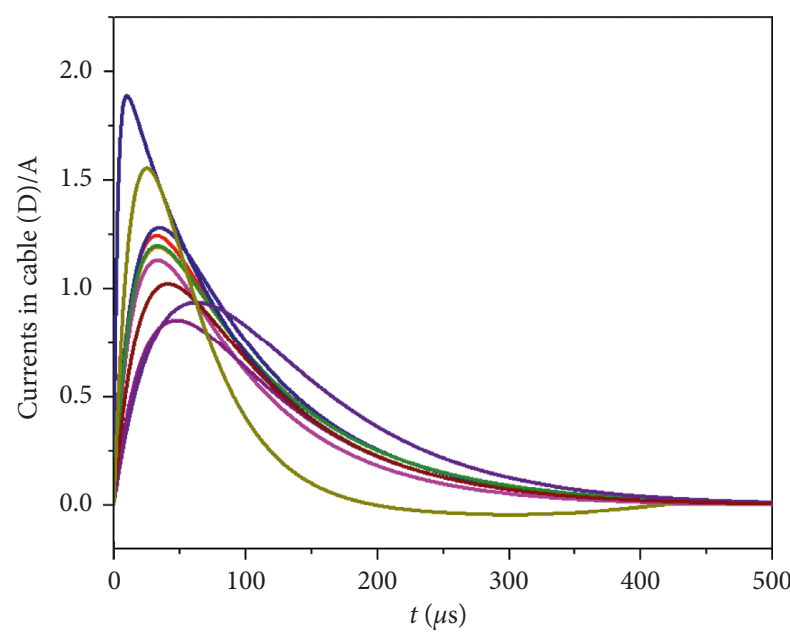

Simplified models

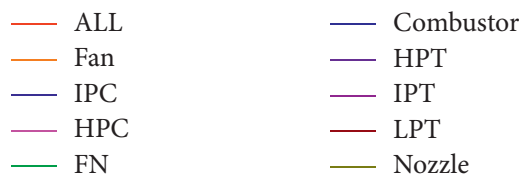

Figure 11: Comparison of simulation results on Cable (D).

time point for the original model after removing the $i$ group of components.

In this paper, the RMSE represents the overall variation of the induced current simulation curve corresponding to the removal of one group in the model and the induced current curve of the original one. The larger the RMSE is, the greater the influence of the component on the induced current of the target cable is. The RMSE is the manifestation of the comprehensive deviation of induction current, so it is the most reliable basis for judging the influence of components on the induced current [17].

The RMSE of the induced current corresponding to the simplified model can be obtained by taking the simulated data into formulation (5) and (6). As shown in Table 3, $\gamma$ represents the RMSE in unit $\mathrm{dB}$.

From Table 3, it can be seen that the error of fuel nozzle and high-pressure turbine are relatively small, which indicates that the two components have less influence on the induced current in the cable, and the error of fan and combustor is relatively large, which indicates that the two components have greater influence on the induced current. Guo [18] has studied the influence of small concave-convex structure in electromagnetic simulation, and the corresponding model simplification scheme was designed. Gutierrez et al. [19] have studied the evaluation criteria of the influence on aeroengine cable induced current. Combining with the investigation results of various scholars, this paper will directly judge the evaluation for the influence of components on induced current according to the combination of investigation results by previous scholars. Combining the above data, a simplification method for the simulation model can be obtained:

(1) Groups with RMSE less than or equal to $1 \mathrm{~dB}$ can be removed directly.

(2) Groups with RMSE greater than $1 \mathrm{~dB}$ and less than $5 \mathrm{~dB}$ can be adjusted structurally, holes with diameter less than $20 \mathrm{~mm}$ can be filled, and microstructures can be smoothed.

(3) Groups with RMSE greater than $5 \mathrm{~dB}$ can only be adjusted slightly. The model is fine-tuned to simplify the structures such as slots, bolts, bulges, and grooves, which are less than $10 \mathrm{~mm}$ in size, and basically maintain the original structure.

\section{Simplification Scheme and Simulation}

According to the simplification method and the simulation data for lightning strike simulation in Table 3 , the aeroengine model is processed as follows:

(1) Because the RMSE of induced current for the removal of fuel nozzle (FN) or high-pressure turbine (HPT) is less than $1 \mathrm{~dB}$, it shows that the effect of these two components on induced currents in cables can be neglected, and the corresponding components can be removed completely.

(2) The RMSE of intermediate-pressure compressor (IPC), high-pressure compressor (HPC), intermediate-pressure turbine (IPT), and low-pressure turbine (LPT) are all more than $1 \mathrm{~dB}$ and less than $5 \mathrm{~dB}$. So, the slots with diameter less than $20 \mathrm{~mm}$ can be filled completely, and the size of the fine structure which is smaller than the mesh size can be flattened.

(3) The RMSE of fan, combustor, and nozzle are much larger than those of other components, so their main structures are all retained, and the fine structures 
TABLE 3: Induced current error for each simplified model.

\begin{tabular}{lcccc}
\hline$\Delta(\mathrm{dB})$ & Cable $(\mathrm{A})$ & Cable $(\mathrm{B})$ & Cable $(\mathrm{C})$ & \\
\hline$\gamma_{\text {Fan }}$ & 12.23 & 1.93 & 2.96 & Cable $(\mathrm{D})$ \\
$\gamma_{\mathrm{IPC}}$ & 4.51 & 2.17 & 2.09 & 1.64 \\
$\gamma_{\mathrm{HPC}}$ & 2.21 & 2.23 & 1.81 & 1.60 \\
$\gamma_{\mathrm{FN}}$ & 0.01 & 0.32 & 0.08 & 1.76 \\
$\gamma_{\text {Combustor }}$ & 0.09 & $\infty$ & 3.81 & 0.01 \\
$\gamma_{\text {HPT }}$ & 0.11 & 0.35 & 0.79 & 1.38 \\
$\gamma_{\text {IPT }}$ & 0.17 & 1.15 & 1.85 & 0.20 \\
$\gamma_{\text {LPT }}$ & 0.10 & 1.59 & 4.11 & 0.99 \\
$\gamma_{\text {Nozzle }}$ & 0.03 & 1.51 & 1.92 & $\infty$ \\
\hline
\end{tabular}

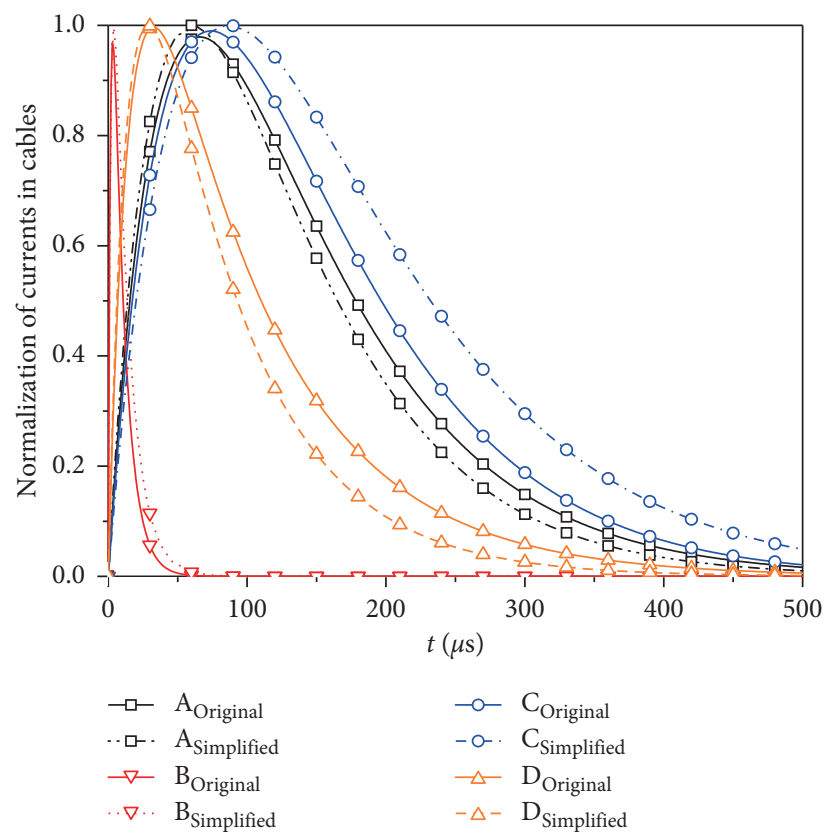

FIGURE 12: Comparison of induced currents between the simplified model and original model.

with sizes less than $10 \mathrm{~mm}$ are smoothed and the holes with diameters less than $10 \mathrm{~mm}$ can be filled.

According to the scheme above, the whole model of the turbofan engine in Section 3.2 is simplified. The induced current curves obtained by the simulations of simplified models are compared with the corresponding results of the original one. Because the maximum value of the induced currents corresponding to different cables are quite different, the simulation results of four cables are normalized, as shown in Figure 12. It can be seen that the variation trends of the curves are the same and the magnitudes are similar. The error parameters are obtained by processing the simulated data as shown in Table 4, and the RMSE are very small. This means that the induced current of the simplified model changes quite little.

However, for the verification of EMC data in modern aeronautical industry, RMSE seems to be a humble standard to measure the data. In 2006, Duffy et al. formally proposed the Feature Selective Validation (FSV) method [20], and the principle of FSV is shown in Figure 13. Subsequently, this
TABLE 4: Errors of the simplified model relative to the original model.

\begin{tabular}{lcccc}
\hline$\Delta(\mathrm{dB})$ & Cable $(\mathrm{A})$ & Cable $(\mathrm{B})$ & Cable $(\mathrm{C})$ & Cable $(\mathrm{D})$ \\
\hline$\gamma_{\text {Original-simplified }}$ & 0.75 & 0.30 & 1.09 & 0.98 \\
\hline
\end{tabular}

method shows a broad application prospect in various fields of computational electromagnetics (CEM) [21]. In recent years, especially with the introduction of the FSV method into the IEEE standard [22] by the working group on computer modeling and simulation verification standards for CEM, the application and expansion of the FSV method in practical engineering have become a hot spot of widespread concern.

The basic principle of the FSV method is to decompose the original data into DC, low-frequency, and high-frequency, and then extract the difference metrics, i.e., amplitude difference measure (ADM) and feature difference measure (FDM), and then form a global difference measure (GDM) [23]. The piecewise approach is shown in Table 5, 


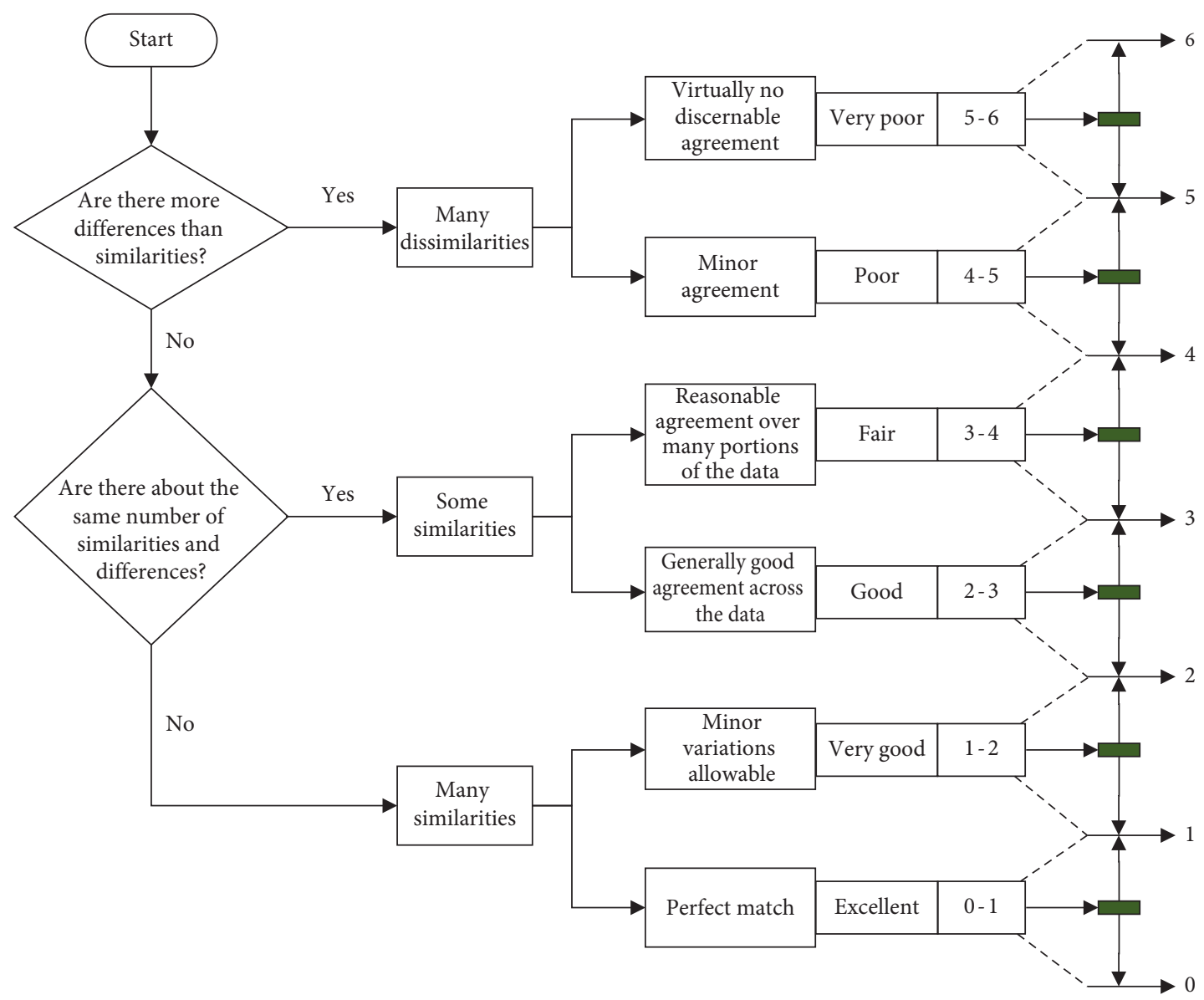

Figure 13: Assessment flow chart.

TABle 5: Piecewise approach.

\begin{tabular}{lcc}
\hline FSV value & Interpretation & Assessment \\
\hline$X \leq 0.1$ & $1+10 * X$ & Excellent \\
$0.1<X \leq 0.2$ & $2+10 *(X-0.099)$ & Very good \\
$0.2<X \leq 0.4$ & $3+5 *(X-0.199)$ & Good \\
$0.4<X \leq 0.8$ & $4+2.5 *(X-0.399)$ & Fair \\
$0.8<X \leq 1.6$ & $5+1.25 *(X-0.799)$ & Poor \\
$X>1.6$ & 6 & Very poor \\
\hline
\end{tabular}

and the "GRADE number" and "SPREAD number" are obtained based on the ADM and FDM. The GRADE number means how many agreement categories are required (starting at excellent) before $85 \%$ of the data. 85 percent of the data fall into the top quality categories, so lower the GRADE number, better the comparison will be. The SPREAD is like a typical standard deviation because it also determines how many categories are needed to include $85 \%$ of the data, but the category is started from the highest rated category (instead of the excellent category, as in GRADE number) [24].

So, the FSV method is applied to the non-normalized data of the simplification simulation results and the original results. The histogram distribution is shown in Figure 14, and the assessments are shown in Table 6, from which it can

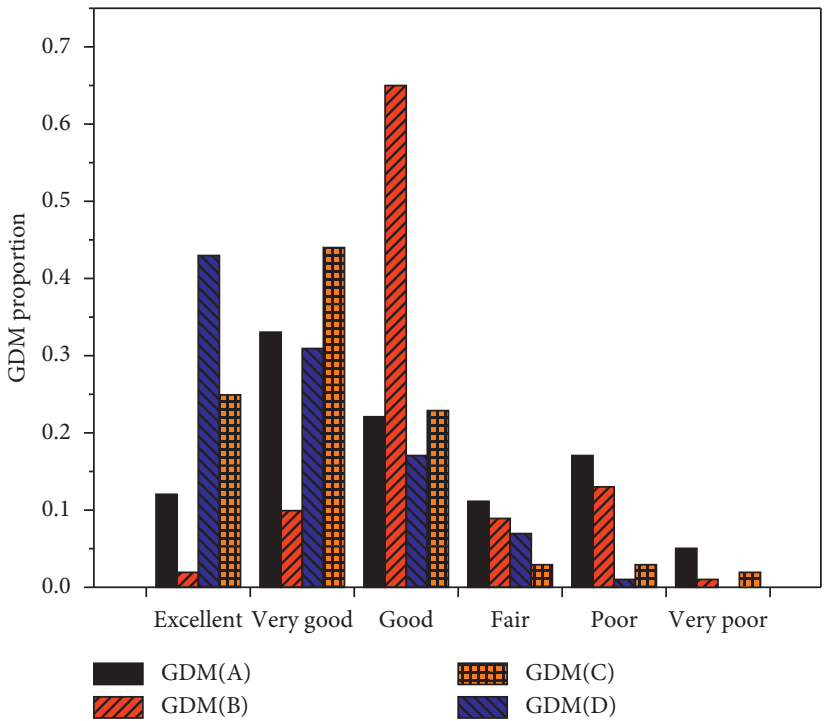

FIGURE 14: Histogram distribution of the GDM obtained from the FSV analysis of data.

be seen that the reliability of the simplified model is more strongly illustrated by the FSV, that is to say, in this lightning strike simulation for induced currents of cables in the 
TABLE 6: FSV results for the comparison.

\begin{tabular}{lccc}
\hline FSV & GDM & GRADE & SPREAD \\
\hline A & 0.281 & 3 & 4 \\
B & 0.349 & 3 & 2 \\
C & 0.123 & 2 & 3 \\
D & 0.208 & 3 & 3 \\
\hline
\end{tabular}

TABle 7: Comparison of computing requirements.

\begin{tabular}{lccc}
\hline Model & Mesh quantity & $\begin{array}{c}\text { Memory } \\
\text { requirement }(\mathrm{G})\end{array}$ & $\begin{array}{c}\text { Computational } \\
\text { time required (h) }\end{array}$ \\
\hline Original & 193904740 & 120 & 158 \\
Simplified & 90090975 & 72 & 49 \\
\hline
\end{tabular}

turbofan engine, the simplification scheme is reasonable and the original model can be replaced.

From Table 7 it can be seen that the number of meshes, memory requirement, and computational time are reduced by $53.5 \%, 40.0 \%$, and $69.8 \%$, respectively. It is obvious that the simplified model not only reduces the requirement of hardware, but also reduces the computing time.

\section{Conclusion}

In this paper, the lightning strike simulation task on a turbofan engine is investigated. The induced currents of the aeroengine controller cables are taken as the simulation objective, and the aeroengine model is simplified by removing components and simplifying the structures. The transmission-line matrix method (TLM) is used to simulate and analyze the effects of different components on the induced currents of cables, and the simulation results are obtained. The error of the simplified simulation scheme is less than $1.3 \mathrm{~dB}$ compared with the original. The computing time and memory requirement are definitely reduced.

Once the simulation target is unequivocal, some components of this kind of large-scale complex device will be redundant. Therefore, the complexity of the model is proportional to the effect of simplification. Further research on simplification of EMC simulation for an aeroengine which is more complex will be needed, which will make this technique more meaningful.

\section{Data Availability}

The data used to support the findings of this study have not been made available because the data in this paper belong to a certain research project which is confidential.

\section{Conflicts of Interest}

The authors declare that they have no conflicts of interest.

\section{Acknowledgments}

This work was supported by the National Natural Science Foundation of China under Grant no 51876089.

\section{References}

[1] S. Rea, D. Linton, E. Orr, and J. McConnell, "Electromagnetic modeling of aircraft air inlet Scoop," in Proceedings of the 33rd European Microwave Conference Proceedings (IEEE Cat. No. 03EX723C), pp. 17-19, Munich, Germany, October 2003.

[2] E. Perrin, F. Tristant, C. Guiffaut, and A. Reineix, "A numerical tool to estimate lightning indirect effects on a composite aircraft," in Proceedings of the 2010 30th International Conference on Lightning Protection (ICLP), pp. 26-28, Cagliari, Italy, September 2010.

[3] J. R. Da Silva and J. P. A. Bastos, "Analysis of power transformer geometry simplifications on electromagnetic and thermodynamic simulations," IEEE Transactions on Magnetics, vol. 51, no. 3, pp. 1-4, 2015.

[4] C. Guiffaut and A. Reineix, "Modeling of different cables types and their combinations for lightning and HIRF EMC studies in the FDTD method," in Proceedings of the 2015 International Conference on Electromagnetics in Advanced Applications (ICEAA), pp. 12-17, Torino, Italy, September 2015.

[5] X. Lu, Research on the Technique of Simplifying the EMC Simulation Model, Xidian University, Xi'an, China, 2009.

[6] A. N. de São José, A. C. P. M. Colin, J. F. Mologni, G. M. Dip, U. D. C. Resende, and S. T. M. Goncalves, "Computational savings based on Three-Dimensional automotive geometries' simplifications in electromagnetics simulations," in Proceedings of the 2013 SBMO/IEEE MTT-S International Microwave \& Optoelectronics Conference (IMOC), pp. 108-111, Rio de Janeiro, Brazil, August 2013.

[7] Z. Reznicek and Z. Raida, "Simplification methodology of complex EM models of small airplanes," in Proceedings of the 2009 International Conference on Electromagnetics in Advanced Applications, pp. 12-19, Torino, Italy, September 2009.

[8] G. G. Gutierrez, D. Mateos Romero, M. R. Cabello et al., "On the design of aircraft electrical structure networks," IEEE Transactions on Electromagnetic Compatibility, vol. 58, no. 2, pp. 401-408, 2016.

[9] Y. She, N. Hou, and K. Yang, "A simulation research of engine control system cables electromagnetic coupling," Aeronautical Computing Technology, vol. 2, no. 48, pp. 66-69, 2018.

[10] Aerospace Recommended Practice, SAE ARP 5416 Aircraft Lightning Test Methods, SAE Aerospace, Warrendale, PA, USA, 2005.

[11] Aerospace Recommended Practice, SAE ARP 5412A Aircraft Lightning Environment and Related Test Waveform, SAE Aerospace, Warrendale, PA, USA, 2005.

[12] L. Liu, Simulation and Research of Lighting Protection on Power Supply System for Flight Test of C919 Airplane, Nanjing University of Aeronautics and Astronautics, Nanjing, China, 2017.

[13] C. Sun and M. Zhang, "Simulation design of harness protection against lightning for aircrafts," in Proceedings of the 2014 International Conference on Lightning Protection (ICLP), pp. 27-34, Shanghai, China, October 2014.

[14] S. Song, C. Gao, Y. Guo, Q. Yang, and B. Zhou, "Study of numerical simulation of aircraft lightning zoning based on CST software," in Proceedings of the 2011 Second International Conference on Mechanic Automation and Control Engineering, pp. 87-90, Inner Mongolia, China, July 2011.

[15] M. Zhang and Z. Huang, "Transient current burst analysis induced in cable harness due to direct lightning strike on aircraft," in Proceedings of the 2010 Asia-Pacific International Symposium on Electromagnetic Compatibility, pp. 34-39, Beijing, China, April 2010. 
[16] B. Michielsen, F. Issac, P. Aguilera, and D. Prost, "Macromodelling of lightning strokes on aircraft," in Proceedings of the 2014 XXXIth URSI General Assembly and Scientific Symposium (URSI GASS), pp. 23-29, Beijing, China, August 2014.

[17] J. Liu, Y. Zhang, J. Lei et al., "Vehicle model simplified method of electromagnetic radiation immunity simulation," Safety and EMC, vol. 1, no. 2, pp. 65-69, 2015.

[18] G. Guo, Research on Simplifying the Stiffeners on Electronic of Electromagnetic Simulations, Xidian University, Xi'an, China, 2014.

[19] G. G. Gutierrez, S. F. Romero, M. Gonzaga et al., "Influence of geometric simplifications on lightning strike simulations," Progress in Electromagnetics Research, vol. 83, pp. 15-32, 2018.

[20] A. P. Duffy, A. J. M. Martin, A. Orlandi, G. Antonini, T. M. Benson, and M. S. Woolfson, "Feature selective validation (FSV) for validation of computational electromagnetics (CEM). Part I-the FSV method," IEEE Transactions on Electromagnetic Compatibility, vol. 48, no. 3, pp. 449-459, 2006.

[21] G. G. Gutierrez, J. Alvarez, E. Pascual-Gil et al., "HIRF virtual testing on the C-295 aircraft: on the application of a pass/fail criterion and the FSV method," IEEE Transactions on Electromagnetic Compatibility, vol. 56, no. 4, pp. 854-863, 2014.

[22] IEEE, IEEE Standard P1597, Standard for Validation of Computational Electromagnetics Computer Modeling and Simulation-Part 1, 2, IEEE, Piscataway, NJ, USA, 2008.

[23] A. Orlandi, A. P. Duffy, B. Archambeault, G. Antonini, D. E. Coleby, and S. Connor, "Feature selective validation (FSV) for validation of computational electromagnetics (CEM). Part II- assessment of FSV performance," IEEE Transactions on Electromagnetic Compatibility, vol. 48, no. 3, pp. 460-467, 2006.

[24] G. Antonini, M. D. Clerico, A. Orlandi, V. Ricchiuti, M. Passacantando, and S. Santucci, "Experimantal characterization and equivalent circuit extraction of nanowires for signal integrity applications," Journal of Pharmacy \& Pharmacology, vol. 17, no. 4, pp. 256-257, 2009. 


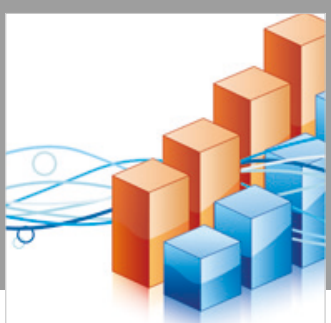

Advances in

Operations Research

\section{-n-m}
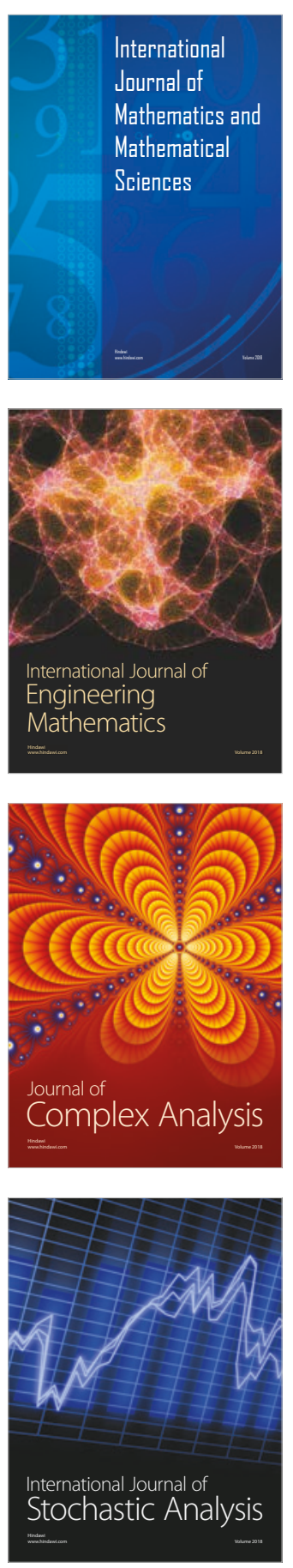
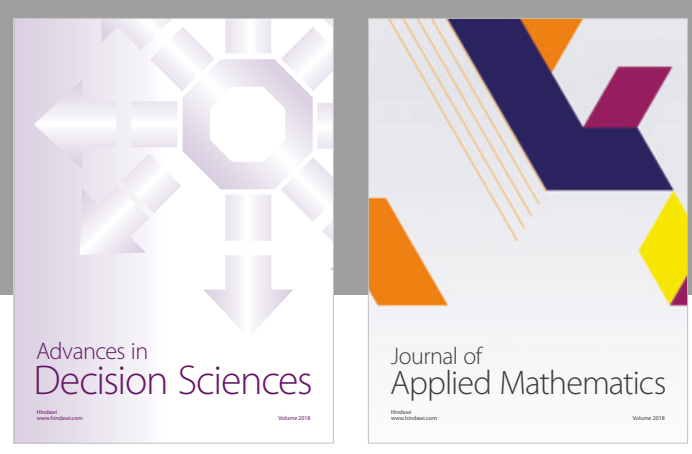

Journal of

Applied Mathematics
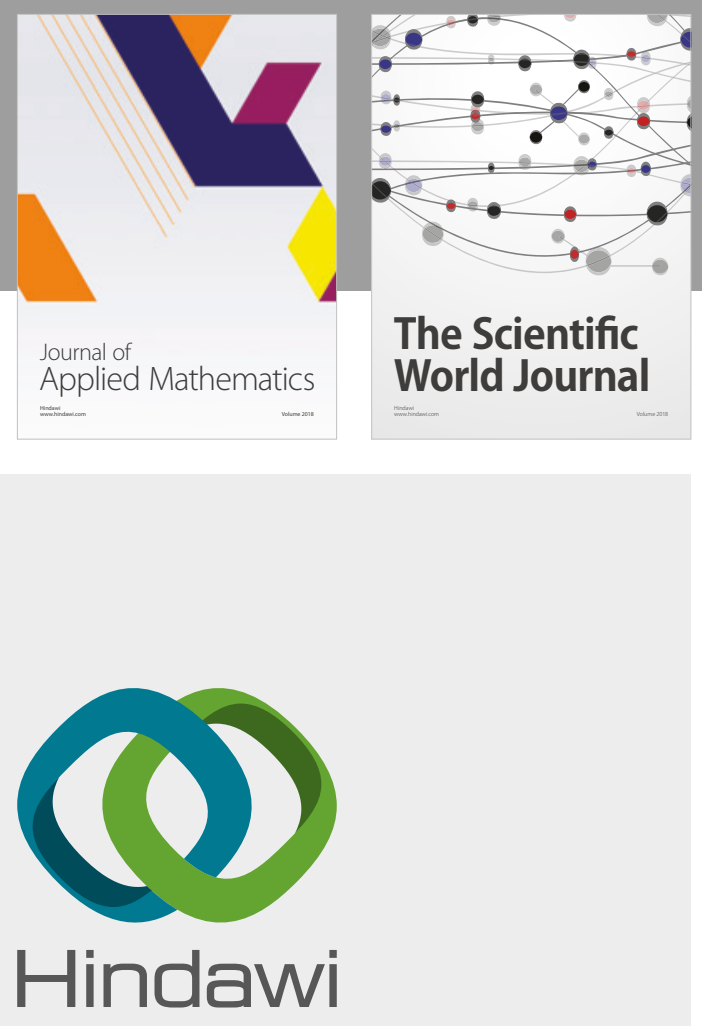

Submit your manuscripts at

www.hindawi.com

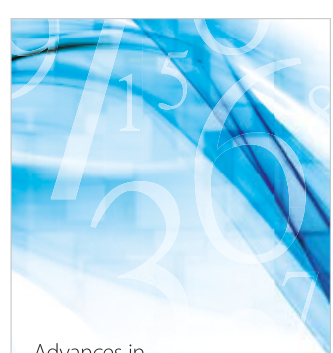

Advances in
Numerical Analysis
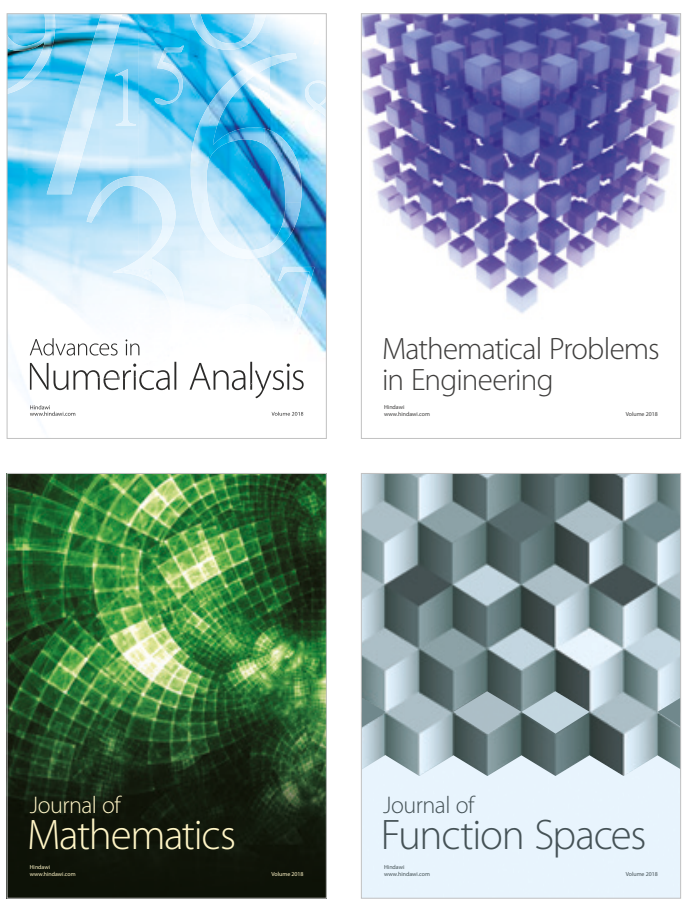

Mathematical Problems in Engineering

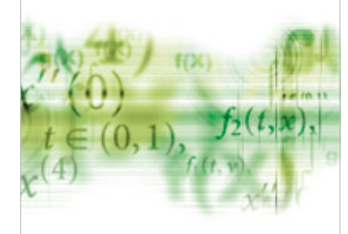

International Journal of

Differential Equations

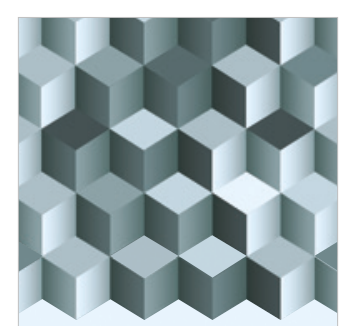

Journal of

Function Spaces

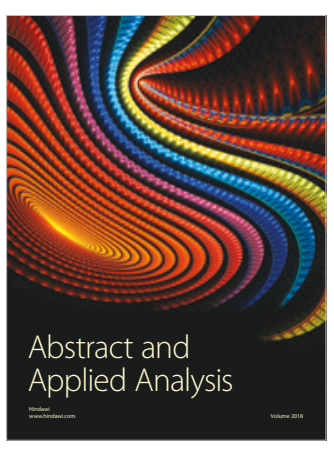

The Scientific

World Journal

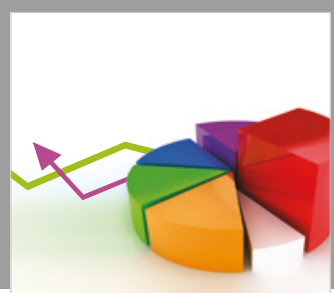

Journal of

Probability and Statistics
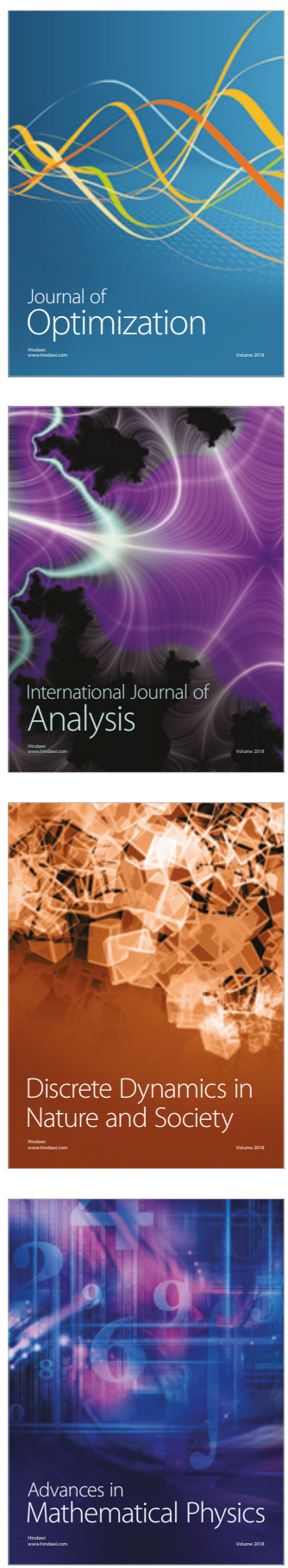